\title{
Microencapsulated conjugated linoleic acid associated with hypocaloric diet reduces body fat in sedentary women with metabolic syndrome
}

\author{
This article was published in the following Dove Press journal: \\ Vascular Health and Risk Management \\ 12 December 2012 \\ Number of times this article has been viewed
}

\author{
Roberta F Carvalho' \\ Sofia K Uehara ${ }^{2}$ \\ Glorimar Rosa ${ }^{1,2}$ \\ 'Medicine Department, Federal \\ University of Rio de Janeiro, Brazil; \\ ${ }^{2}$ Nutrition and Dietetic Department, \\ Josué de Castro Institute of Nutrition, \\ Federal University of Rio de Janeiro, \\ Brazil
}

Background: Animal studies have suggested beneficial effects of conjugated linoleic acid (CLA) in reducing body fat mass and improvement in the serum lipid profile and glycemia. However, these effects are controversial in humans. The purpose of this study was to investigate the effects of microencapsulated CLA supplementation on body composition, body mass index, waist circumference, and blood pressure in sedentary women with metabolic syndrome.

Methods: This study was a placebo-controlled and randomized clinical trial. Fourteen women diagnosed with metabolic syndrome received light strawberry jam enriched or not with microencapsulated CLA (3 g/day) as a mixture of $38.57 \%$ cis- 9 , trans -11 , and $39.76 \%$ trans -10 , cis-12 CLA isomers associated with a hypocaloric diet for 90 days. The subjects were monitored to assess variables associated with the metabolic syndrome, in addition to assessing adherence with the intervention.

Results: There were no significant effects of microencapsulated CLA on the lipid profile or blood pressure. Mean plasma insulin concentrations were significantly lower in women supplemented with microencapsulated CLA $\left(\Delta \mathrm{T}_{90}-\mathrm{T}_{0}=-12.87 \pm 4.26 \mu \mathrm{U} / \mathrm{mL}, P=0.02\right)$. Microencapsulated CLA supplementation did not alter the waist circumference, but there was a reduction in body fat mass detected after 30 days $(\Delta=-2.68 \% \pm 0.82 \%, P=0.02)$, which was maintained until the 90 -day intervention period $(\Delta=-3.32 \% \pm 1.41 \%, P=0.02)$ in the microencapsulated CLA group. The placebo group showed this effect only after 90 days $(\Delta=-1.97 \% \pm 0.60 \%, P=0.02)$, but had a reduced waist circumference $\left(\Delta \mathrm{T}_{90}-\mathrm{T}_{0}=-4.25 \pm 1.31 \mathrm{~cm}, P=0.03\right)$.

Conclusion: Supplementation with mixed-isomer microencapsulated CLA may have a favorable effect on glycemic control and body fat mass loss at an earlier time in sedentary women with metabolic syndrome, although there were no effects on lipid profile and blood pressure.

Keywords: conjugated linoleic acid, metabolic syndrome, body composition, cardiovascular disease

\section{Introduction}

Metabolic syndrome is a complex disorder that is showing an increasing prevalence in the population worldwide. ${ }^{1}$ It is represented by a set of cardiovascular risk factors commonly associated with central adiposity. In addition to abdominal obesity, the major components of metabolic syndrome are hypertension, glucose intolerance, hypertriglyceridemia, and reduced serum high-density lipoprotein cholesterol. ${ }^{2}$ Functional foods have occupied a remarkable place in dietary therapies. ${ }^{3}$ At present, functional lipids such as conjugated linoleic acid (CLA) have attracted considerable attention. ${ }^{4}$ 
CLA is a mixture of isomers of linoleic acid formed by fermentation in the animal rumen in relatively small quantities, and is present in meat and dairy products. ${ }^{5}$ Among the isomers, cis-9, trans-11, trans-10, and cis-12 are the most studied $^{6}$ through the use of commercial CLA, and they have a different isomer profile to that found in natural foods, which have the cis-9, trans-11 isomer in the greatest amount. ${ }^{7}$

Among the processes developed to increase the stability of commercial CLA, microencapsulation provides the necessary protection because it coats the lipid with an encapsulating matrix, producing smaller particles, which have demonstrated greater efficiency in digestive absorption. ${ }^{8}$ There are no studies evaluating the effects of CLA provided in a microencapsulated formulation in humans.

It has been postulated that CLA has beneficial effects in reducing body fat mass and it has been shown to improve the lipid profile and glycemia in animal studies. ${ }^{9}$ Nevertheless, results from studies in humans that have evaluated the effects of CLA on these parameters are controversial, with some showing beneficial effects ${ }^{10,11}$ and others showing negative effects. ${ }^{12,13}$ Thus, randomized controlled clinical trials are needed to evaluate the efficacy and safety of orally ingested CLA isomers.

This study aimed to investigate the influence of a hypocaloric diet combined with microencapsulated CLA supplementation on biochemical, clinical, anthropometric, and body composition variables in sedentary women with metabolic syndrome. We hypothesized that microencapsulated CLA supplementation would improve glucose metabolism, the serum lipid profile, and body composition, and decrease other cardiovascular risk factors associated with the metabolic syndrome.

\section{Materials and methods Experimental design}

The volunteers were informed about the procedures that would be performed and signed an informed consent form. The study was approved by the ethics committee on research at Clementino Fraga Filho University Hospital (068/07), and included 17 sedentary adult women aged 30-50 years recruited through advertisements in widely circulated newspapers and posters in places with a heavy throughput of people.

The volunteers were instructed to consume $15 \mathrm{~g}$ of light strawberry jam daily for 90 days, supplied in $240 \mathrm{~g}$ pots with a standard-sized spoon, as part of a randomized, placebo-controlled clinical trial. The jam was prepared by the researchers and was either enriched with microencapsulated
CLA or not enriched (placebo). The volunteers were randomly distributed into two groups, one receiving placebo and the other receiving microencapsulated CLA supplementation.

Participants were assessed before and during the 90-day intervention period, with consultations every 2 weeks for anthropometric, body composition, and clinical evaluation. Blood samples were taken monthly for biochemical assessment. A supply of light strawberry jam was given to the volunteers at each consultation.

\section{Eligibility criteria}

The volunteers selected were diagnosed with the metabolic syndrome according to International Diabetes Federation criteria, ${ }^{14}$ were obese (body mass index $30.0-34.9 \mathrm{~kg} / \mathrm{m}^{2}$ ), sedentary, nonsmoking, nondiabetic, not post-menopausal, not having a previous hypocaloric diet, and not taking dietary supplements or any medication to reduce body weight. Women consuming alcohol above recommended levels (15 g/day), those using drugs to control glycemia or dyslipidemia, and those who were pregnant or lactating were excluded.

\section{Nutritional intervention}

The method used to microencapsulate CLA involved complex coacervation to obtain a paste. The sugar content in the strawberry jam was reduced by $30 \%$. Subjects received $3 \mathrm{~g} /$ day of microencapsulated (MiKron microcapsules, São Paulo, Brazil) CLA (Idealfarma, São Paulo, Brazil) containing $38.57 \%$ of cis-9, trans-11 isomers, and $39.76 \%$ of trans10, cis-12 isomers, in an equal proportion (50:50). Placebo was in the form of light strawberry jam not enriched with microencapsulated CLA.

The hypocaloric diet was prescribed in accordance with the dietary habits of volunteers and the recommendations for individuals with metabolic syndrome, ${ }^{15}$ aiming at reducing body weight by about $2 \mathrm{~kg}$ per month. Adherence to the dietary plan was checked at fortnightly consultations by analysis of food records containing details of dietary intake and number of spoonfuls of jam taken daily. As an additional measure of adherence, participants were asked to return containers with any remaining jam at the end of each supplementation period. Food records were evaluated using Food Processor software version 12 with adaptation to the reality of the Brazilian food database (Esha Research, 1984, Salem, MA).

\section{Physical activity}

The subjects did not alter their physical activity during the study, which was verified by a questionnaire ${ }^{16}$ applied before 
and after the intervention period. Exercise was assessed as metabolic equivalents of task (METs). ${ }^{17}$ Subjects were considered to be sedentary if they did not do physical exercise or exercised with a caloric expenditure below 3 METs at a frequency of less than twice a week.

\section{Blood sample collection and biochemical evaluation}

Blood samples were collected into vacuum tubes without anticoagulant after overnight fasting for 12 hours. The samples were centrifuged at $4500 \mathrm{rpm}$ for 15 minutes in order to obtain the serum, and subsequently were separated into aliquots placed in Eppendorf tubes and stored at $-20^{\circ} \mathrm{C}$ until biochemical analysis.

Blood glucose was measured using the glucose oxidase/ peroxidase method ${ }^{18}$ (BioSystems commercial kit, Paraná, Brazil) using the A15 auto-analyzer (BioSystems SA). Insulinemia was analyzed using a chemiluminescence kit (ImmuChem ${ }^{\mathrm{TM}}$ coated tube, MP Biomedical, Irvine, CA). Insulin resistance was estimated using the homeostasis model assessment of insulin resistance index (HOMA-IR). ${ }^{19}$ The HOMA index was calculated using the formula: [fasting glucose $(\mathrm{mg} / \mathrm{dL}) \times 0.0555 \times$ fasting insulin $(\mu \mathrm{U} / \mathrm{mL})] / 22.5$.

Analysis of serum concentrations of triglycerides, total cholesterol, and high-density lipoprotein cholesterol were performed using a commercial enzymatic colorimetric method (BioSystems) with an A15 auto-analyzer, using the glycerol phosphate oxidase/peroxidase method ${ }^{20}$ to determine triglycerides. Cholesterol oxidase/peroxidase method ${ }^{21}$ was used for determining total cholesterol and direct detergent ${ }^{22}$ for determining high-density lipoprotein cholesterol. Low-density lipoprotein cholesterol values were obtained using the Friedewald, Levy, and Fredrickson formula. ${ }^{23}$

\section{Clinical assessments of body and anthropometric composition}

Blood pressure was measured using an aneroid sphygmomanometer (Bournemouth International Centre obese adult cuff $38 \times 16 \mathrm{~cm}$ model) and a stethoscope (Littman Cardiology, São Paulo, Brazil) by the auscultation method. ${ }^{24}$ The assessment of body fat mass was performed by tetrapolar electrical bioimpedance (Biodynamics 450 model, Seattle, WA), according to the recommendations reported by Heyward and Stolarczyk. ${ }^{25}$ Body weight was measured using an electronic scale (maximum capacity of $150 \mathrm{~kg}$, accurate to 100 g, Filizola, São Paulo, Brazil) and height was assessed using a stadiometer (accurate to $1 \mathrm{~mm}$, Standard Sanny ${ }^{\circledR}$, São Paulo, Brazil ${ }^{26}$ for calculation of body mass index. ${ }^{27}$
Waist circumference was measured using an inextensible and inelastic tape measure (accurate to $0.1 \mathrm{~cm}$, Standard Sanny ${ }^{\circledR}$, São Paulo, Brazil) at the midpoint between the rib cage and iliac crest. ${ }^{28}$

\section{Statistical analysis}

All statistical analyses were conducted using the Statistical Package for Social Sciences version 16(SPSS Inc, Chicago, IL). Results were presented as the mean \pm standard error of the mean. Normality of the variables was tested by the Shapiro-Wilk test. For nonparametric variables we used the Mann-Whitney test to assess differences between groups and the Wilcoxon signed ranks test to assess changes in each group. For variables with a normal distribution, we used the Student's $t$-test for independent samples to compare the means between groups and the Student's $t$-test for paired samples to assess changes in each group, and evaluating those changes throughout the study. A $P$ value $<0.05$ was considered to be statistically significant.

\section{Results}

One of the volunteers in the microencapsulated CLA group withdrew before the end of the study due to epigastric pain and a request from her physician that she should discontinue using the experimental jam. This effect was deemed to be unrelated to use of microencapsulated CLA, given that this patient reported that she had had this disorder prior to the study. No other adverse effect was reported by any of the participants. Two participants from the placebo group were lost to follow-up. The volunteers who left the study showed the same characteristics as those who completed it. Fourteen women completed the study, including seven (50\%) from the CLA group and seven (50\%) from the placebo group.

At the beginning of the study, there was no statistically significant difference between dietary, physical activity, biochemical, clinical, anthropometric, and body composition variables between the groups (Tables 1-3). Characteristics of the sedentary women in the study population are shown in Table 1. The volunteers maintained their usual caloric expenditure, without undertaking physical exercise. Volunteers adhered to their prescribed dietary plan, with a significant decrease in total caloric intake at day $90(1976.8 \pm 67.31 \mathrm{kcal}$, $P=0.03$, in the CLA group; $1745.5 \pm 118.20 \mathrm{kcal}, P=0.05$, in the placebo group). During the 3 months of this study, reversal of the metabolic syndrome was observed in four women in each study group, according to International Diabetes Federation criteria. ${ }^{14}$ 
Table I Baseline characteristics of the sedentary female study population (mean \pm SEM)

\begin{tabular}{lcc}
\hline & ME CLA $(\mathbf{n}=\mathbf{7})$ & Placebo $(\mathbf{n}=\mathbf{7})$ \\
\hline Age (years) & $40.0 \pm 5.23$ & $42.0 \pm 1.91$ \\
Height $(\mathrm{m})$ & $1.63 \pm 0.02$ & $1.60 \pm 0.03$ \\
Exercise $(\mathrm{kcal} /$ day) & $22.27 \pm 1.59$ & $23.35 \pm 1.6$ \\
Energy intake $(\mathrm{kcal} /$ day) & $2120.8 \pm 50.11$ & $2042.6 \pm 51.96$ \\
\hline
\end{tabular}

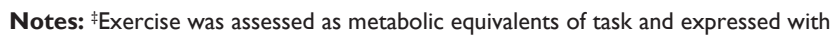
$\mathrm{kcal} /$ day. There was no statistical difference between the ME CLA and placebo groups.

Abbreviations: ME CLA, microencapsulated conjugated linoleic acid; SEM, standard error of the mean.

Results of the biochemical analysis can be found in Table 2. There was no significant effect of microencapsulated CLA supplementation on glycemia. Insulin values decreased significantly in the microencapsulated CLA group at day 90 $(P=0.02)$. In the placebo group, there was a trend towards decreasing values $(P=0.09)$. There were no significant differences in insulin concentrations between the treatment groups. HOMA-IR did not change significantly between groups, and the effect of this variable before and after treatment in both

Table 2 Biochemical assessment of the volunteers before and during the intervention period (mean \pm SEM)

\begin{tabular}{|c|c|c|c|}
\hline & $\mathbf{T}_{0}$ & $\mathbf{T}_{90}$ & $\underline{\text { Value }}$ \\
\hline & & & $\Delta\left(\mathrm{T}_{90}-\mathrm{T}_{0}\right)$ \\
\hline \multicolumn{4}{|c|}{ Fasting glucose $(\mathrm{mg} / \mathrm{dL})$} \\
\hline ME CLA & $92.57 \pm 7.92$ & $86.43 \pm 5.20$ & $-6.14 \pm 3.99$ \\
\hline Placebo & $84.00 \pm 3.15$ & $86.00 \pm 2.82$ & $2.00 \pm 2.65$ \\
\hline \multicolumn{4}{|c|}{ Insulin $(\mu \mathrm{U} / \mathrm{mL})$} \\
\hline ME CLA & $46.04 \pm 5.65$ & $33.16 \pm 4.45^{*}$ & $-12.87 \pm 4.26^{*}$ \\
\hline Placebo & $45.99 \pm 4.87$ & $35.31 \pm 4.29$ & $-10.67 \pm 5.44$ \\
\hline \multicolumn{4}{|c|}{ HOMA-IR } \\
\hline ME CLA & $8.83 \pm 1.19$ & $6.86 \pm 0.77$ & $-1.97 \pm 1.17$ \\
\hline Placebo & $9.66 \pm 1.26$ & $7.46 \pm 0.98$ & $-2.20 \pm 1.38$ \\
\hline \multicolumn{4}{|c|}{ Triglycerides (mg/dL) } \\
\hline ME CLA & $157.00 \pm 2.74$ & $128.86 \pm 1.23$ & $-28.14 \pm 21.38$ \\
\hline Placebo & $136.7 \mid \pm 2.09$ & $|3| .43 \pm 4.1 \mid$ & $-5.28 \pm 31.45$ \\
\hline \multicolumn{4}{|c|}{ Total cholesterol (mg/dL) } \\
\hline ME CLA & $|83.43 \pm| 5.5 \mid$ & $165.86 \pm 11.41$ & $-17.57 \pm 10.40$ \\
\hline Placebo & $179.14 \pm 11.30$ & $\mid 85.43 \pm 9.01$ & $6.28 \pm 12.46$ \\
\hline \multicolumn{4}{|c|}{ LDL cholesterol (mg/dL) } \\
\hline ME CLA & $|83.43 \pm| 5.5 \mid$ & $93.57 \pm 1.07$ & $-9.85 \pm 7.30$ \\
\hline Placebo & $98.89 \pm 9.14$ & $107.29 \pm 6.90$ & $8.4 \pm 10.29$ \\
\hline \multicolumn{4}{|c|}{ HDL cholesterol (mg/dL) } \\
\hline ME CLA & $48.57 \pm 3.50$ & $46.57 \pm 2.53$ & $-2.00 \pm 2.77$ \\
\hline Placebo & $52.86 \pm 6.58$ & $5 \mathrm{I} .7 \mathrm{I} \pm 5.27$ & $-1.14 \pm 6.44$ \\
\hline
\end{tabular}

Notes: *Statistical differences in each group compared with baseline $(P<0.05)$; there was no statistical difference between the ME CLA and placebo groups; ${ }^{\ddagger}$ Calculated as [(fasting glucose $\left.(\mathrm{mg} / \mathrm{dL}) \times 0.0555\right) \times$ fasting insulin $\left.(\mathrm{mU} / \mathrm{mL})\right] / 22.5$. Abbreviations: ME CLA, microencapsulated conjugated linoleic acid; HOMA-IR, homeostasis model assessment - insulin resistance; LDL, low-density lipoprotein; $\mathrm{HDL}$, high-density lipoprotein; SEM, standard error of the mean; $\mathrm{T}_{90}$, day 90 ; $\mathrm{T}_{0}$, baseline. the microencapsulated CLA group and the placebo group did not change significantly during the study. There were no significant changes observed in the components of the lipid profile between the groups before and after treatment.

Clinical, anthropometric, and body composition data are presented in Table 3 . There were no statistically significant differences in systolic or diastolic blood pressure between the groups and within each group throughout the study. In the microencapsulated CLA group, the percentage of body fat mass decreased significantly after day $30(P=0.02)$, day $60(P=0.02)$ and day $90(P=0.02)$, whereas in the placebo group, a significant decrease was found only at day $90(P=0.02)$. There was no significant difference in this regard between the groups. The microencapsulated CLA group showed an earlier loss of body fat mass in comparison with the placebo group (Figure 1). There were no significant changes in body weight or body mass index in the placebo and CLA groups, although we observed an average reduction during the study. There was also no significant difference in body weight and body mass index between the treatment groups. Waist circumference decreased significantly in the placebo group at day $30(P=0.03)$, day $60(P=0.03)$, and day $90(P=0.02)$. There was no significant difference in waist circumference between the groups.

\section{Discussion}

The present study found that microencapsulated CLA supplementation significantly reduced plasma insulin concentrations. This change was also observed in the placebo group, although it was not statistically significant. This may be attributable to changes in body composition in both groups. Furthermore, microencapsulated CLA supplementation was associated with decreases in glycemia and insulin resistance, but these were not statistically significant. This could be due to the fact that subjects participating in the study were nondiabetic and the study variables may not be as sensitive to changes in extreme ranges. ${ }^{29}$ Moreover, the microencapsulated formulation of CLA enabled oxidative stability that could improve the study variables more than studies that have used the purified form of CLA. Other studies have shown either no interference ${ }^{30,31}$ or harmful effects on glucose metabolism. ${ }^{32,33}$ Some studies have found higher glycemia and insulin resistance with use of CLA, ${ }^{12,32,34}$ and the change is more significant in studies using the trans 10 cis 12 isomer. ${ }^{12,32}$

The lipid profile did not change significantly with microencapsulated CLA supplementation in this study. These findings are similar to results from other studies. ${ }^{35-37}$ Moreover, 
Table 3 Clinical, anthropometric, and body composition evaluations in both groups before and during the intervention period (mean \pm SEM)

\begin{tabular}{|c|c|c|c|c|c|c|c|}
\hline & \multirow[t]{2}{*}{$\mathbf{T}_{0}$} & \multirow[t]{2}{*}{$\mathbf{T}_{30}$} & \multirow[t]{2}{*}{$\mathbf{T}_{60}$} & \multirow[t]{2}{*}{$\mathbf{T}_{90}$} & \multicolumn{3}{|l|}{ Value } \\
\hline & & & & & $\Delta\left(T_{30}-T_{0}\right)$ & $\Delta\left(\mathrm{T}_{60}-\mathrm{T}_{0}\right)$ & $\Delta\left(\mathrm{T}_{90}-\mathrm{T}_{0}\right)$ \\
\hline \multicolumn{8}{|c|}{$\overline{\text { SBP }(\mathrm{mmHg})}$} \\
\hline ME CLA & $117.14 \pm 3.59$ & $121.43 \pm 4.04$ & $118.57 \pm 1.43$ & $120.00 \pm 3.78$ & $4.28 \pm 3.68$ & $1.42 \pm 4.04$ & $2.85 \pm 5.21$ \\
\hline Placebo & $\mathrm{I} \mid 8.57 \pm 3.40$ & || $4.29 \pm 4.8 \mid$ & $112.14 \pm 4.06$ & $|I 5.7| \pm 5.28$ & $-4.28 \pm 5.71$ & $-6.42 \pm 3.22$ & $-2.85 \pm 5.65$ \\
\hline \multicolumn{8}{|c|}{ DBP $(\mathrm{mmHg})$} \\
\hline ME CLA & $77.14 \pm 1.84$ & $78.57 \pm 4.04$ & $78.57 \pm 2.61$ & $79.26 \pm 4.14$ & $\mathrm{I} .42 \pm 3.40$ & $1.42 \pm 3.40$ & $2.14 \pm 4.61$ \\
\hline Placebo & $80.71 \pm 3.17$ & $80.00 \pm 3.09$ & $72.86 \pm 3.59$ & $80.00 \pm 3.09$ & $-0.71 \pm 4.14$ & $-7.85 \pm 3.42$ & $-0.7 \mathrm{I} \pm 4.42$ \\
\hline \multicolumn{8}{|l|}{ BF (\%) } \\
\hline ME CLA & $35.95 \pm 1.27$ & $33.23 \pm 2.04 *$ & $33.90 \pm 1.60^{*}$ & $32.63 \pm 1.91 *$ & $-2.68 \pm 0.82 *$ & $-2.20 \pm 0.67 *$ & $-3.32 \pm \mid .4 I^{*}$ \\
\hline Placebo & $38.06 \pm 0.68$ & $37.36 \pm 0.90$ & $36.56 \pm 1.00$ & $36.09 \pm 0.95^{*}$ & $-0.70 \pm 0.60$ & $-|.50 \pm 0.7|$ & $-1.97 \pm 0.60^{*}$ \\
\hline \multicolumn{8}{|c|}{ Weight (kg) } \\
\hline ME CLA & $86.91 \pm 3.67$ & $85.99 \pm 3.72$ & $85.49 \pm 4.06$ & $84.97 \pm 4.11$ & $-0.92 \pm 0.65$ & $-1.42 \pm 1.06$ & $-1.94 \pm 1.09$ \\
\hline Placebo & $82.90 \pm 2.99$ & $81.07 \pm 3.17$ & $80.86 \pm 3.50$ & $80.41 \pm 3.47$ & $-1.82 \pm 0.36$ & $-2.01 \pm 0.99$ & $-2.48 \pm 1.31$ \\
\hline \multicolumn{8}{|c|}{ BMI $\left(\mathbf{k g} / \mathrm{m}^{2}\right)$} \\
\hline ME CLA & $32.53 \pm 0.77$ & $32.18 \pm 0.80$ & $31.98 \pm 0.98$ & $31.78 \pm 1.01$ & $-0.35 \pm 0.25$ & $-0.55 \pm 0.4 \mathrm{I}$ & $-0.74 \pm 0.43$ \\
\hline Placebo & $32.30 \pm 0.80$ & $31.56 \pm 0.83$ & $31.51 \pm 1.01$ & $31.33 \pm 1.06$ & $-0.74 \pm 0.17$ & $-0.78 \pm 0.41$ & $-0.97 \pm 0.55$ \\
\hline \multicolumn{8}{|c|}{$W C(\mathrm{~cm})$} \\
\hline ME CLA & $99.9 \pm 2.98$ & $99.64 \pm 2.56$ & $99.11 \pm 3.61$ & $97.53 \pm 3.63$ & $-0.25 \pm 0.88$ & $-0.78 \pm 1.57$ & $-2.37 \pm 1.34$ \\
\hline Placebo & $97.64 \pm 3.42$ & $95.21 \pm 3.68^{*}$ & $94.00 \pm 3.12^{*}$ & $93.39 \pm 2.70 *$ & $-2.42 \pm 0.82^{*}$ & $-3.64 \pm 1.28 *$ & $-4.25 \pm 1.31 *$ \\
\hline
\end{tabular}

Notes: *Statistical differences in each group compared with baseline $(P<0.05)$. There was no statistical difference between the ME CLA and placebo groups $(P<0.05)$. Abbreviations: ME CLA, microencapsulated conjugated linoleic acid; SEM, standard error of the mean; SBP, systolic blood pressure; DBP, diastolic blood pressure; BF, body fat; BMI, body mass index; WC, waist circumference; $T_{30}$, day $30 ; T_{60}$, day $60 ; T_{90}$, day $90 ; T_{0}$, baseline.

the effects of CLA on the lipid profile are controversial due to the difference in dosages of CLA used, proportions of CLA isomers, and population samples. Some studies showed an increase $^{34}$ and others a reduction in high-density lipoprotein cholesterol, ${ }^{12,38,39}$ and a reduction in total cholesterol, lowdensity lipoprotein cholesterol, ${ }^{11}$ and triglycerides ${ }^{40}$ with CLA supplementation.

There was no significant change in blood pressure with microencapsulated CLA supplementation. Raff et $\mathrm{al}^{41}$ and Sluijs et al ${ }^{42}$ evaluated the effects of CLA supplementation with an equivalent amount of isomer mixtures in individuals with normal weight and in obese subjects, respectively, and detected no change in blood pressure levels. On the other hand, some studies have found a significant decrease in blood pressure in groups on CLA supplementation. ${ }^{37,43}$

In this study, both treatment groups showed a decrease in body fat mass, with no significant differences between groups. However, these results were observed at an earlier time in the microencapsulated CLA group, with significant changes after 30 days in contrast with the placebo group, in which the changes occurred only after 90 days. It is important to consider that early reduction of body fat provides people with encouragement to continue on a hypocaloric diet and develop healthy lifestyle habits.

The current study confirmed previous observations indicating body fat mass reduction with CLA supplementation. ${ }^{44,45}$
Gaullier et $\mathrm{al}^{11}$ observed a decrease in body fat mass by supplementation with an isomeric mixture of CLA at doses of $3.4 \mathrm{~g} /$ day in subjects without dietary restriction for 24 months, with a significant reduction in body fat mass only in the first 6 months. After this period, the placebo group showed the same results, suggesting an adaptation mechanism or a decrease in the effectiveness of CLA supplementation. However, the positive results seen with the supplemental CLA mixture were observed only after 3 months. On the other hand, some studies did not show a reduction in body fat mass. ${ }^{29,35,46}$

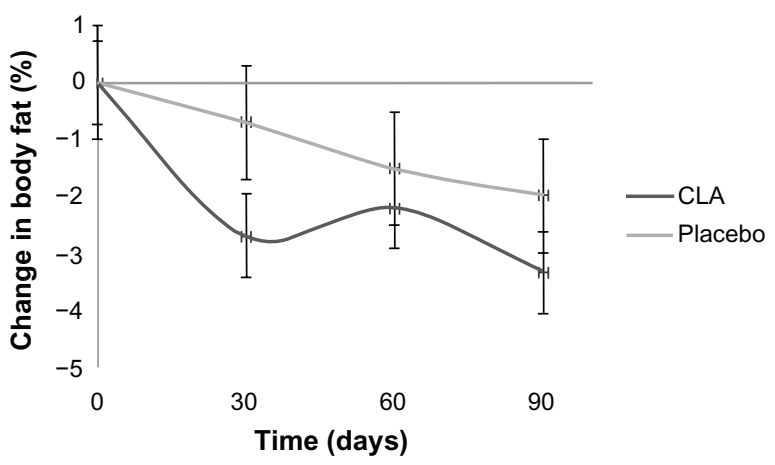

Figure I Percentage change in body fat in subjects administered placebo or microencapsulated CLA during 90 days.

Notes: Values are means with $95 \%$ confidence intervals $(n=14)$ and were measured at the same time points for all two groups (ie, day 0, 30,60 and 90). Intervals not including 0 are significant in the CLA group and only at day 90 in the placebo group.

Abbreviation: CLA, conjugated linoleic acid. 
Among the mechanisms by which CLA decreases body fat, there is one that may be validated, ie, CLA prevents lipid accumulation in human adipocytes by acting as a modulator of peroxisome proliferator-activated receptor gamma. ${ }^{47,48}$

In the present study, waist circumference did not show any significant change between groups, but decreased significantly in the placebo group from day 30 , even though both groups achieved a reduction in body fat mass. These findings are in accordance with those of other studies reporting that CLA supplementation promotes reduction of body fat mass, mostly in areas other than the abdomen, despite that fact that CLA has a tendency to decrease the amount of abdominal fat mass. ${ }^{13,44}$ In this way, women with a gynoid fat distribution will probably benefit from use of CLA. Although microencapsulated CLA reduced body fat mass in our study, it did not cause a significant reduction in body weight. This result is similar to that found in other studies. . $^{30,42,46}$

Subjects from both groups showed remission of components of the metabolic syndrome, underscoring the importance of a hypocaloric diet in the treatment of this condition. ${ }^{49}$ The effects of CLA supplementation on blood pressure and biochemical variables suggest that microencapsulated CLA supplementation in women with metabolic syndrome for 3 months is safe. Only female subjects were chosen for this study due to gender-related differences in body composition. ${ }^{25}$ Because we evaluated body composition as an outcome variable, we aimed to homogenize the study sample. Moreover, Chen et $\mathrm{al}^{39}$ showed a better effect of CLA supplementation in lowering body mass index in female subjects than in male subjects.

The limitation of this study is that the sample size was small due to the strict inclusion and exclusion criteria. On the other hand, this is the only study that has evaluated the effects of microencapsulated CLA in women with metabolic syndrome.

\section{Conclusion}

The findings of the present study suggest that, in sedentary women with metabolic syndrome, supplementation with microencapsulated CLA $3 \mathrm{~g} /$ day for 3 months improves glycemic control and has a significant effect in decreasing body fat early on. Further, in our study, there was no change in serum lipids or blood pressure. At the same time, it is important to highlight that more studies are needed to demonstrate further the factors that may modulate the effects observed in this study.

\section{Acknowledgments}

We are particularly grateful to the Carlos Chagas Filho Foundation for Research Support for its financial assistance and to Idealfarma for donating CLA and MiKron microcapsules for microencapsulating the CLA used in this study.

\section{Disclosure}

The authors report no personal financial interests in this work.

\section{References}

1. Athyros VG, Ganotakis ES, Elisaf MS, Liberopoulos EN, Goudevenos IA, Karagiannis A. Prevalence of vascular disease in metabolic syndrome using three proposed definitions. Int J Cardiol. 2007;117:204-210.

2. Alberti KG, Eckel RH, Grundy SM, Zimmet PZ, Cleeman JI, Donato KA. Harmonizing the metabolic syndrome: a joint interim statement of the International Diabetes Federation Task Force on Epidemiology and Prevention; National Heart, Lung, and Blood Institute; American Heart Association; World Heart Federation; International Atherosclerosis Society; and International Association for the Study of Obesity. Circulation. 2009;120:1640-1645.

3. National Cholesterol Education Program; National Heart, Lung and Blood Institute; National Institutes of Health. Third Report of the National Cholesterol Education Program (NCEP) Expert Panel on Detection, Evaluation, and Treatment of High Blood Cholesterol in Adults (Adult Treatment Panel III). Circulation. 2006;106:3143-3421.

4. Yanagita T, Nagao K. Functional lipids and the prevention of the metabolic syndrome. Asia Pac J Clin Nutr. 2008;17 Suppl 1:189-191.

5. Or-Rashid MM, Wright TC, McBride BW. Microbial fatty acid conversion within the rumen and the subsequent utilization of these fatty acids to improve the healthfulness of ruminant food products. Appl Microbiol Biotechnol. 2009;84:1033-1043.

6. Churruca I, Fernández-Quintela A, Portillo MP. Conjugated linoleic acid isomers: differences in metabolism and biological effects. Biofactors. 2009;35:105-111.

7. Taylor CG, Zahradka P. Dietary conjugated linoleic acid and insulin sensitivity and resistance in rodent models. Am J Clin Nutr. 2004; 79 Suppl 6:S1164-S1168.

8. Lee JS, Song YB, Lee JY, Kim MK, Jun SJ, Lee HG. Optimization and oxidative stability of the microencapsulated conjugated linoleic acid. Int J Biol Macromol. 2009;45:348-351.

9. Aminot-Gilchrist DV, Anderson HDI. Insulin resistance-associated cardiovascular disease: potential benefits of conjugated linoleic acid. Am J Clin Nutr. 2004;79 Suppl 6:S1159-S1163.

10. Pfeuffer M, Fielitz K, Laue C, et al. CLA does not impair endothelial function and decreases body weight as compared with safflower oil in overweight and obese male subjects. J Am Coll Nutr. 2011;30:19-28.

11. Gaullier JM, Halse J, Høye K, et al. Supplementation with conjugated linoleic acid for 24 months is well tolerated by and reduces body fat mass in healthy, overweight humans. J Nutr. 2005;135:778-784.

12. Risérus U, Arner P, Brismar K, Vessby B. Treatment with dietary trans10cis12 conjugated linoleic acid causes isomer-specific insulin resistance in obese men with the metabolic syndrome. Diabetes Care. 2002;25:1516-1521.

13. Taylor JS, Williams SR, Rhys R, James P, Frenneaux MP. Conjugated linoleic acid impairs endothelial function. Arterioscler Thromb Vasc Biol. 2006;26:307-312.

14. International Diabetes Federation. IDF Worldwide Definition of the Metabolic Syndrome. International Diabetes Federation. [serial on the Internet]. 2005. http://www.idf.org/home. Accessed July 28, 2006.

15. Brazilian Society of Cardiology. First Brazilian guidelines for diagnosis and treatment of metabolic syndrome. Arq Bras Cardiol. 2005; 84 Suppl 1:3-28.

16. Gomes VB, Siqueira KS, Sichieri R. Physical activity among a random sample of the Rio de Janeiro. Cad Saude Publica. 2001;17:969-976. Portuguese.

17. Ainsworth BE, Haskell WL, Herrmann SD, et al. 2011 Compendium of Physical Activities: a second update of codes and MET values. Med Sci Sports Exerc. 2011;43:1575-1581. 
18. Trinder R. Determination of glucose in blood using glucose with alternative oxygen acceptor. Ann Clin Biochem. 1969;6:24-27.

19. Matthews DR, Hosker JP, Rudenski BA, Naylor DF, Treacher DF, Turner RC. Homeostasis model assessment: insulin resistance and $\beta$-cell function from fasting plasma glucose and insulin concentrations in man. Diabetologia. 1985;28:412-419.

20. Bucolo G, David H. Quantitative determination of serum triglycerides by the use of enzymes. Clin Chem. 1973;19:476-482.

21. Allain CC, Poon LS, Chan CS, Richmond W, Fu PC. Enzymatic determination of total serum cholesterol. Clin Chem. 1974;20:470-475.

22. Warnick GR, Nauck M, Rifai N. Evolution of methods for measurement of HDL-cholesterol: from ultracentrifugation to homogeneous assays. Clin Chem. 2001:47:1579-1596.

23. Friedewald WT, Levy RI, Fredrickson DS. Estimation of the concentration of low-density lipoprotein cholesterol in plasma, without use of the preparative ultracentrifuge. Clin Chem. 1972;18:499-502.

24. Pickering TG, Hall JE, Appel LJ, Falkner BE, Graves J, Hill MN. Recommendations for blood pressure measurement in humans and experimental animals: part 1: blood pressure measurement in humans a statement for professionals from the Subcommittee of Professional and Public Education of the American Heart Association Council on High Blood Pressure Research. Circulation. 2005;111:697-716.

25. Heyward VH, Stolarczyk KM. Fundamentals of body composition. In: Heyward VH, Stolarczyk KM, editors. Body Composition Assessment Applied. São Paulo, Brazil: Manole; 2000.

26. Gibson RS. Principles of Nutritional Assessment. 2nd ed. New York, NY: Oxford University Press Inc; 2005.

27. World Health Organization. Obesity: Preventing and Managing the Global Epidemic: Report of a WHO Consultation on Obesity. Geneva, Switzerland: World Health Organization; 1998.

28. World Health Organization. Measuring Obesity - Classification and Description of Anthropometric Data. Report of a WHO Regional Office Consultation on the Epidemiology of Obesity. Copenhagen, Denmark: World Health Organization; 1988.

29. Lambert EV, Goedecke JH, Bluett K, et al. Conjugated linoleic acid versus high-oleic acid sunflower oil: effects on energy metabolism, glucose tolerance, blood lipids, appetite and body composition in regularly exercising individuals. Br J Nutr. 2007;97:1001-1011.

30. Raff M, Tholstrup T, Basu S, Nonboe P, Sørensen MT, Straarup EM. A diet rich in conjugated linoleic acid and butter increases lipid peroxidation but does not affect atherosclerotic, inflammatory, or diabetic risk markers in healthy young men. J Nutr. 2008;138:509-514.

31. Brown AW, Trenkle AH, Beitz DC. Diets high in conjugated linoleic acid from pasture-fed cattle did not alter markers of health in young women. Nutr Res. 2011;31:33-41.

32. Ingelsson E, Risérus U. Effects of trans10cis12CLA-induced insulin resistance on retinol-binding protein 4 concentrations in abdominally obese men. Diabetes Res Clin Pract. 2008;82:e23-e24.

33. Iwata T, Kamegai T, Yamauchi-Sato Y, et al. Safety of dietary conjugated linoleic acid (CLA) in a 12-weeks trial in health overweight Japanese male volunteers. J Oleo Sci. 2007;56:517-525.

34. Moloney F, Yeow TP, Mullen A, Nolan JJ, Roche HM. Conjugated linoleic acid supplementation, insulin sensitivity, and lipoprotein metabolism in patients with type 2 diabetes mellitus. Am J Clin Nutr. 2004;80:887-895.
35. Joseph SV, Jacques H, Plourde M, Mitchell PL, McLeod RS, Jones PJ. Conjugated linoleic acid supplementation for 8 weeks does not affect body composition, lipid profile, or safety biomarkers in overweight, hyperlipidemic men. J Nutr. 2011;141:1286-1291.

36. Laso N, Brugué E, Vidal J, et al. Effects of milk supplementation with conjugated linoleic acid (isomers cis- 9 , trans- 11 and trans-10, cis-12) on body composition and metabolic syndrome components. Br J Nutr. 2007:98:860-867.

37. Aryaeian N, Shahram F, Djalali M, et al. Effect of conjugated linoleic acid, vitamin $\mathrm{E}$ and their combination on lipid profiles and blood pressure of Iranian adults with active rheumatoid arthritis. Vasc Health Risk Manag. 2008;4:1423-1432.

38. Racine NM, Watras AC, Carrel AL, et al. Effect of conjugated linoleic acid on body fat accretion in overweight or obese children. Am J Clin Nutr. 2010;91:1157-1164.

39. Chen SC, Lin YH, Huang HP, Hsu WL, Houng JY, Huang CK. Effect of conjugated linoleic acid supplementation on weight loss and body fat composition in a Chinese population. Nutrition. 2012;28:559-565.

40. Noone EJ, Roche HM, Nugent AP, Gibney MJ. The effect of dietary supplementation using isomeric blends of conjugated linoleic acid on lipid metabolism in healthy human subjects. Br J Nutr. 2002;88:243-251.

41. Raff M, Tholstrup T, Sejrsen K, Straarup EM, Wiinberg N. Diets rich in conjugated linoleic acid and vaccenic acid have no effect on blood pressure and isobaric arterial elasticity in healthy young men. $J$ Nutr. 2006;136:992-997.

42. Sluijs I, Plantinga Y, de Roos B, Mennen LI, Bots ML. Dietary supplementation with cis-9, trans-11 conjugated linoleic acid and aortic stiffness in overweight and obese adults. Am J Clin Nutr. 2010;91: 175-183.

43. Zhao WS, Zhai JJ, Wang YH, et al. Conjugated linoleic acid supplementation enhances antihypertensive effect of ramipril in Chinese patients with obesity-related hypertension. Am J Hypertens. 2009;22: 680-686.

44. Gaullier JM, Halse J, Høivik HO, et al. Six months supplementation with conjugated linoleic acid induces regional-specific fat mass decreases in overweight and obese. Br J Nutr. 2007;97:550-560.

45. Norris LE, Collene AL, Asp ML, et al. Comparison of dietary conjugated linoleic acid with safflower oil on body composition in obese postmenopausal women with type 2 diabetes mellitus. Am J Clin Nutr. 2009;90:468-476.

46. Nazare JA, de la Perrière AB, Bonnet F, et al. Daily intake of conjugated linoleic acid-enriched yoghurts: effects on energy metabolism and adipose tissue gene expression in healthy subjects. $\mathrm{Br} J$ Nutr. 2007;97:273-280.

47. Kennedy A, Chung S, LaPoint K, Fabiyi O, McIntosh MK. Trans-10, cis-12 conjugated linoleic acid antagonizes ligand-dependent PPARgamma activity in primary cultures of human adipocytes. $J$ Nutr. 2008; 138:455-461.

48. Granlund L, Juvet LK, Pedersen JI, Nebb HI. Trans10, cis12-conjugated linoleic acid prevents triacylglycerol accumulation in adipocytes by acting as a PPAR gamma modulator. J Lipid Res. 2003;44:1441-1452.

49. Amati L, Marzulli G, Martulli M, Chiloiro M, Jirillo E. Effects of a hypocaloric diet on obesity biomarkers: prevention of low-grade inflammation since childhood. Curr Pharm Des. 2010;16:893-897.
Vascular Health and Risk Management

\section{Publish your work in this journal}

Vascular Health and Risk Management is an international, peerreviewed journal of therapeutics and risk management, focusing on concise rapid reporting of clinical studies on the processes involved in the maintenance of vascular health; the monitoring, prevention and treatment of vascular disease and its sequelae; and the involvement of

\section{Dovepress}

metabolic disorders, particularly diabetes. This journal is indexed on PubMed Central and MedLine. The manuscript management system is completely online and includes a very quick and fair peer-review system, which is all easy to use. Visit http://www.dovepress.com/ testimonials.php to read real quotes from published authors. 